\title{
Semi-automatic building footprint using multirotor and fixed wing UAV
}

\author{
Nabila Ismail, Khairul Nizam Tahar \\ Centre of Studies for Surveying Science and Geomatics, Faculty of Architecture, Planning and Surveying, \\ Universiti Teknologi MARA, Malaysia
}

\begin{tabular}{l} 
Article Info \\
\hline Article history: \\
Received Jan 10, 2019 \\
Revised May 1, 2019 \\
Accepted Jul 1, 2019 \\
\hline
\end{tabular}

Keywords:

Accuracy

Building

Extraction

Footprints

UAV

\begin{abstract}
The current techniques to extract building footprints frequently involve Light Detection and Ranging (LiDAR), as well as satellite images. The UAV is proposed to acquire aerial imagery in this study as it is more efficient, time-efficient, and inexpensive, especially for small budget projects. Hence, this study evaluated the building footprints from different UAV platforms. The objectives of this study are to perform the different UAV image processing of building footprints using fixed-wing UAV and multi-rotor UAV, as well as to analyse the outcomes of building footprints from different UAV platforms with existing layout plans. The methodology begins with a preliminary study, and continued with acquisition of data via UAV, data processing, and lastly, analyses of the outcome from different platforms. Based on the analysis in terms of area, the accuracy gained by using multi rotor UAV had been better than that for fixed wing UAV with area difference of $13.648 \mathrm{~m}^{2}$ compared to $18.091 \mathrm{~m}^{2}$. Lastly, it can be summarised that the area displayed better results with multi rotor UAV, in comparison to the area obtained by using fixed wing UAV. Thus, it is recommended for future studies to use other UAV platforms and other methods for extraction of building footprints, such as orthoimages and digital elevation model.
\end{abstract}

Copyright $@ 2020$ Institute of Advanced Engineering and Science. All rights reserved.

\section{Corresponding Author:}

Khairul Nizam Tahar,

Centre of Studies for Surveying Science and Geomatics,

Faculty of Architecture, Planning and Surveying,

Universiti Teknologi MARA,

40450 Shah Alam, Selangor, Malaysia.

Email: nizamtahar@gmail.com

\section{INTRODUCTION}

Building footprints are very important data in many fields, such as in updating the cadastral database, quality checking for existing footprint or map, city-based planning, DEM (digital elevation model) acquisition, vegetation monitoring, telecommunication, and 3D city modelling [1]. Town planning department requires latest information regarding the specific features at the development area. One of the important feature is building footprint. The building footprint will determine the design of the new development area. Therefore, the fast building footprint data acquisition is very important to speed up the planning stage [2-3]. The development of automated solutions of building footprints has increased in order to speed up the process, as well as to reduce the costs of the process. Some works that use aerial and satellite are able to detect buildings and delineate their footprints by using images and exploiting shadows or by generating DSM or by using multi-spectral information [4]. Nowadays, the current techniques to extract building footprints frequently involve Light Detection and Ranging (LiDAR) and satellite images, which are mostly done in manual and rely largely on architectural blueprints or skilled modellers [5]. As in LiDAR technique, certain aspects are not correctly removed due to 'debris' or small building being left out during the extraction process [6]. Vegetation or tree that 'interferes' with the urban features is also a problem in 
extracting building footprints. Thus, it is recommended for the vegetation and trees to be selected and removed during the pre-processing stage [7]. As in satellite images, the accuracy of the technique is affected with the measured shadows and the surrounding environments.

Conventionally, the manned aircraft is used to capture aerial images, but now, many projects are accomplished by using UAVs [8-9]. The advantage of aerial photogrammetry, it reduces field work, for example, when certain data are absent or the information has to be re-evaluated, it can be done in the office using the photograph, which makes the aerial photogrammetry faster as there is no need to perform field work [10]. Other than that, it is more efficient to map a large area by using aerial photogrammetry than the traditional survey methods [11]. UAV photogrammetry is a new photogrammetric measurement tool. UAV photogrammetry has discovered numerous new applications in the close range that introduce an inexpensive option to the conventionally-manned aerial photogrammetry [12-13]. The image quality is better than the satellite imagery as it is located at $100,000 \mathrm{~km}$ from the surface of the earth. Satellite images has different resolutions based on its specific sensors [14]. UAV images can provide small ground sampling distance compared to satellite images [15]. Nowadays, UAVs can be used as a particular, automatic and computer-controlled data acquisition and measurement platform, with the latest developments of economical sensors, including off-the-shelf virtual cameras, GPS / INS (Global Positioning System device / Inertial Navigation System device), navigation unit, and laser scanner [16].

In the geomatics field, UAV systems and their applications have been vastly investigated regarding their regulations and data processing [17]. Typical image-based aerial surveying with an UAV platform needs flight mission planning and ground control points (GCPs) measurement for geo-referencing functions. After that, the images can be used for stitching and mosaicking purposes or they can be used as input for a photogrammetric process [18]. The final products can be used for the production of orthoimages, 3D modelling applications, or for the extraction of further metric information. The different image processing technique might cause the accuracy of photogrammetric results. There are a lot of commercial software available in the open market. The user need to determine the correct technique to achieve the desired results [19]. The different align photo and build dense algorithm applied during photogrammetric image processing will effect the end products. Therefore, this study investigates the accuracy of different align photo and build dense during photogrammetric image processing specifically for building footprints [20].

In order to obtain more accurate results, some researchers used the fusion of different data sources, for example, synthetic aperture radar (SAR) with optical image fusion or LiDAR with optical image fusion [21]. Other than that, the manual techniques in obtaining the building footprints are labour intensive, slow, and expensive. Therefore, the UAV is proposed to acquire aerial imagery in this study as it is more efficient, time-saving, and inexpensive, especially for small budget projects. This study evaluated building footprints from different UAV platforms.

\section{RESEARCH METHOD}

The methodology is divided into four phases. Phase 1 consists of preliminary study, including site reconnaissance. Site Reconnaissance was carried out at buildings with perfect geometry for validation purposes. This location is also suitable for UAV studies because it has sufficient space for UAV launching and landing. This phase also covers the selection hardware and software for the study. Phantom 3 Professional was used for image acquisition for multi rotor UAV, while eBee was applied for fixed wing $\mathrm{UAV}$, and other hardware used had been laptop, mobile phone, and transmitter as shown in Figure 1. It is important to determine the condition of the hardware prior to image acquisition so as to avoid any damage or system failure during the flight mission.
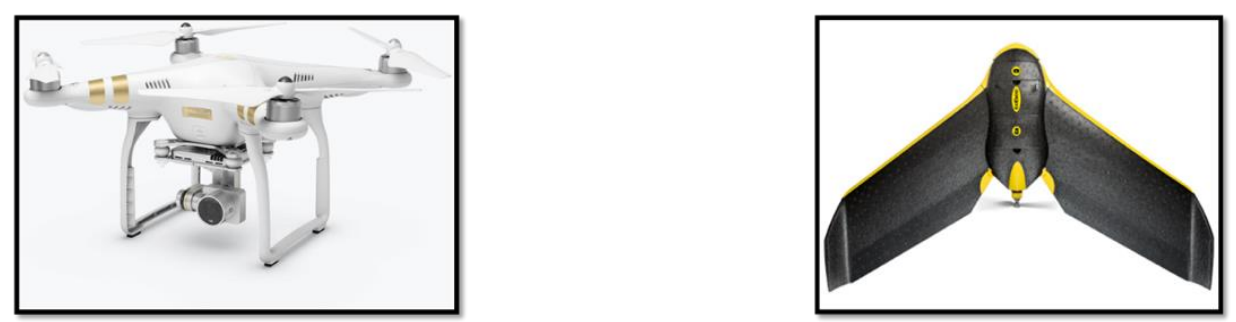

Figure 1. Phantom 3 Professional [22] and eBee [23] 
All acquired images were processed using Agisoft software. Agisoft PhotoScan is a photogrammetric software solution for automatic generation of dense point clouds, textured polygonal models, georeferenced true orthomosaics, and DSMs/DTMs from images. It allows rapid processing with highly accurate results. Agisoft PhotoScan is capable of processing thousands of photos, yet all the processing is performed locally, without the need to transmit the data outside the company [24]. Phase 2 refers to data collection that includes camera calibration, UAV calibration, and flight planning. Camera calibration is the important part that was performed prior to flight planning. The camera was calibrated in order to assess the condition of the camera before data collection. Thus, the reliability of the camera was determined. In this study, two types of UAV were used to capture the images at the study area. All factors were considered because any problem that occurs during image acquisition would lead to poor images that cannot be used for data processing. The PhotoModeler software was used to perform the camera calibration process.

A self-camera calibration was conducted to carry out the camera configuration that can determine the position of the main principle point, the main range focal length, and the optical distortion coefficients. The process began with the calibration sheet printed from the software that was standardised with the calibration point on a piece of paper with a certain size. The procedure for Phantom 3 Pro IMU calibration took between 5 and 10 minutes. The Phantom 3 Pro should not be moved at all during the IMU calibration process, otherwise the procedure would fail. If the IMU is out of sync, this procedure may need to be done more than once. Hence, the Phantom 3 Pro was placed on a flat surface. Next, spirit or bubble level was employed to check for a nice flat surface. The flight planning is an important stage, which required planning after the reconnaissance stage. The condition of the study area was explored before the flight mission in order to find the best location for the hovering and landing of UAV during the flight mission. The location of hovering and landing usually requires 10 meter radius [25].

In this study, the Altizure was used to create the flight planning for image capture. The objective of the flight planning was to determine the total number of flight lines and to determine the amount of time needed to fly and to capture the entire study area. The flight planning is an important aspect that is related to cost and time. The altitude was set at $100 \mathrm{~m}$, while the overlap was $70 \%$, and the side lap was $50 \%$. Phase 3 covers data processing where all the acquired images were processed. This process is also known as postprocessing. The UAV raw images were downloaded into a computer after a flight mission was completed. The quality of images was verified prior to the processing stage. Some of the images might have some quality issues, such as blurred images and colour balancing error, which can be caused during flight mission. Agisoft PhotoScan Software was used to process the images, and three levels of accuracy could be opted from the Align Photos (AP), which are high, medium, and low. Other than that, five levels of accuracy are available in Build Dense Cloud (BDC), which are ultra-high, high, medium, low, and lowest. Every level of accuracy was employed in this study to observe the effect on the results. A total of thirty UAV images were processed using Agisoft PhotoScan software. Figure 2 illustrates the UAV image processing which highlights the different methods in align photo and to build dense cloud.

All images obtained during data acquisition were imported to Agisoft PhotoScan software. Then, automatically, the images were set in their position because the images were geotagged with the coordinates during image acquisition. All images were self-oriented after importing them into this software. After the images were imported, the images had to be aligned and continued with Place Markers for Ground Control Points (GCP) and Verification Points (VP) to be marked in the images. The GCPs were used to determine the position of its aerial photo image in the coordinate system. VPs were used to check on the accuracy assessment of photogrammetric results of the study area. Next, the analyses were preceded with Build Dense Cloud, Build Mesh, Build Texture, Generate DSM, and Generate Orthophoto.

As the DSM, DTM, and orthophoto were obtained from the Agisoft PhotoScan Software, the results were processed by using ArcGIS for building extraction. From the generated DSM and DTM, the parameters considered to distinguish the building from other features had been the height and the area. Non-building features and all other short features were eliminated. Smaller features that may be extracted as buildings were resolved by filtering the extracted features according to its area. All the preliminary values considered in all the extractions had been based on the characteristics of the buildings and structures built in the study area, thus reflecting the actual observation. The final step of the building extraction refers to the simplification of the polygons extracted to eliminate irregular-shaped features as shown in Figure 3. Phase 4 covers data analysis of building footprints using fixed wing UAV and multi rotor UAV, while the outcomes of building footprints from different UAV platforms with existing layout plans were analysed. The outcomes of building footprints were evaluated in terms of area. 


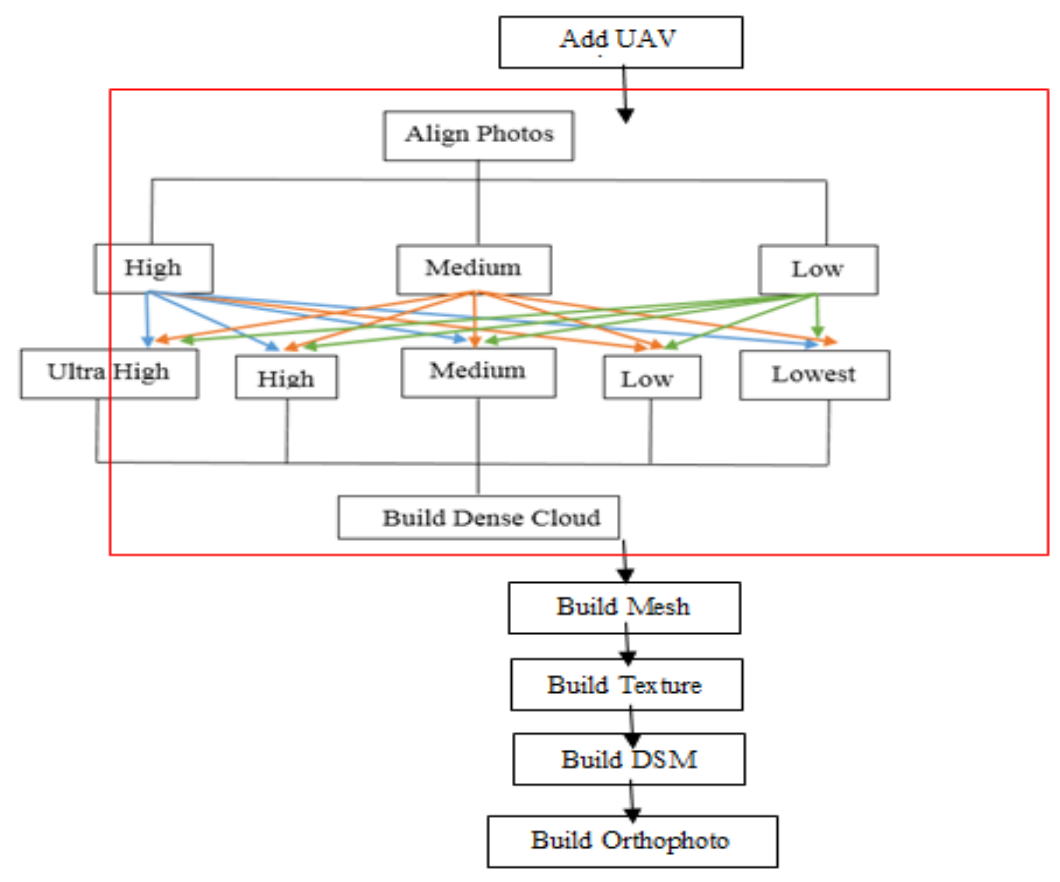

Figure 2. Processing for different method in align photo and build dense cloud
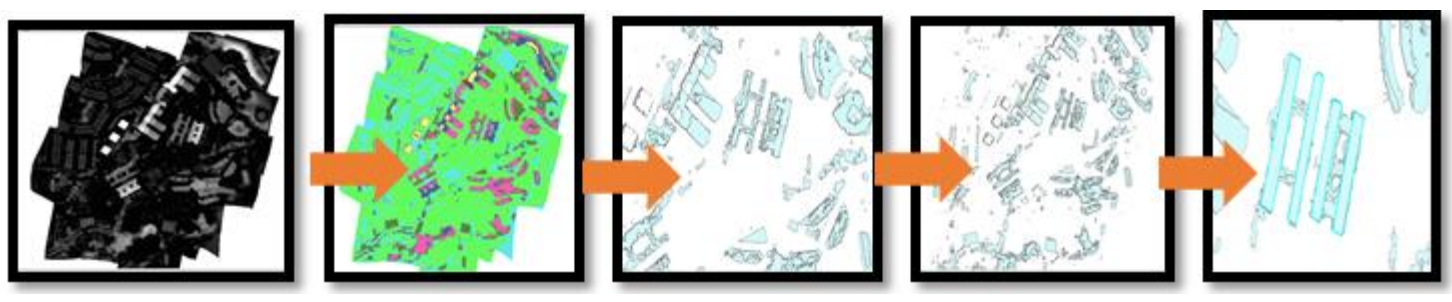

Figure 3. NDSM-Extract by Height-Extract by Area-Raster to Vector-Building Simplification

\section{RESULTS AND ANALYSIS}

There are DTM, DSM, and Orthophoto as the results from each time the UAV images were processed in Agisoft PhotoScan Software. The results from Agisoft PhotoScan Software, such as DTM, DSM, and orthophoto, were processed in ArcGIS to extract the buildings. The actual data for this study had been based on the existing layout plans, which was the area of the buildings. The comparison of area between Multi Rotor UAV and Fixed Wing UAV was analysed into three main align photos: high align, medium align, and low align. Each main align photo had five different build dense accuracy levels. The result for high, medium and low align photo for five different build dense accuracy levels is shown in Figure 4.

Figure 4 shows the high, medium and low align photo for all build dense cloud almost have similar pattern except lowest build dense cloud represents a few spikes. The high align photo shows the consistent graph for all samples while the medium and low align photo show the inconsistencies of the results. The differences for all samples between multi rotor and fixed wing UAV illustrate in Table 1.

Table 1 shows the large different for high and medium align for most build dense cloud in all samples. It is because the multi rotor and fixed wing UAV platform produced the different results which effect the extraction of building footprint. The different for low align records small different except for several samples. The results for high and medium align show the maximum RMSE value in multi rotor and fixed wing UAV appear to be the lowest build dense, while the minimum RMSE value in ultra-high build dense. The result for low align shows the maximum RMSE value in multi rotor UAV appears to be the lowest build dense, while the minimum RMSE value in medium build dense. Other than that, the maximum RMSE value in fixed wing UAV seems to be the lowest build dense, while the minimum RMSE value in ultra-high build dense as shown in Figure 5. 

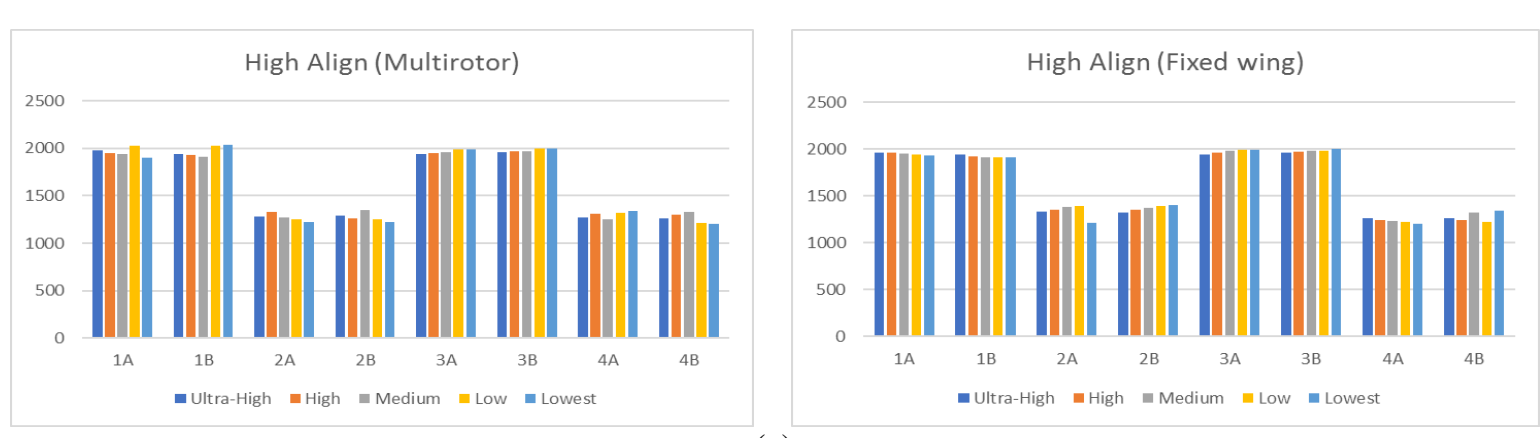

(a)
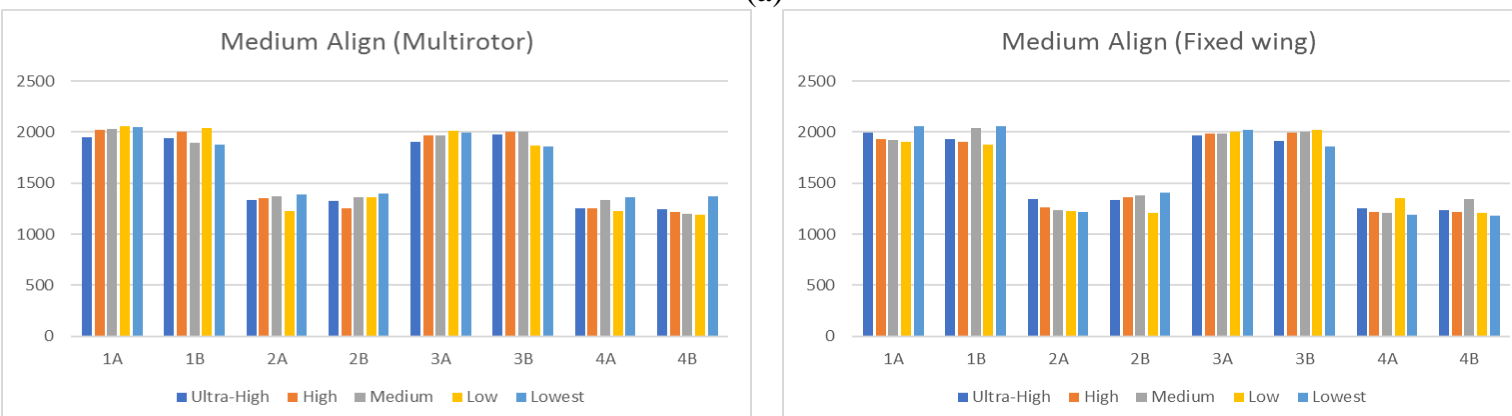

(b)
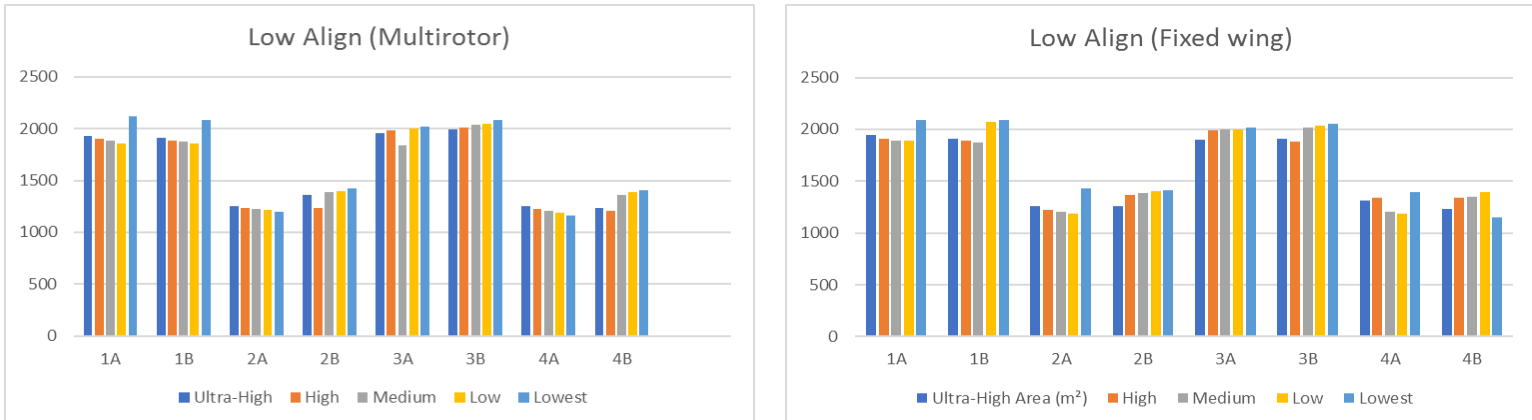

(c)

Figure 4. Results; a) high align photo, b) medium align photo, c) low align photo

Table 1. Different between Multi Rotor and Fixed Wing UAV for High Align Photo (Area)

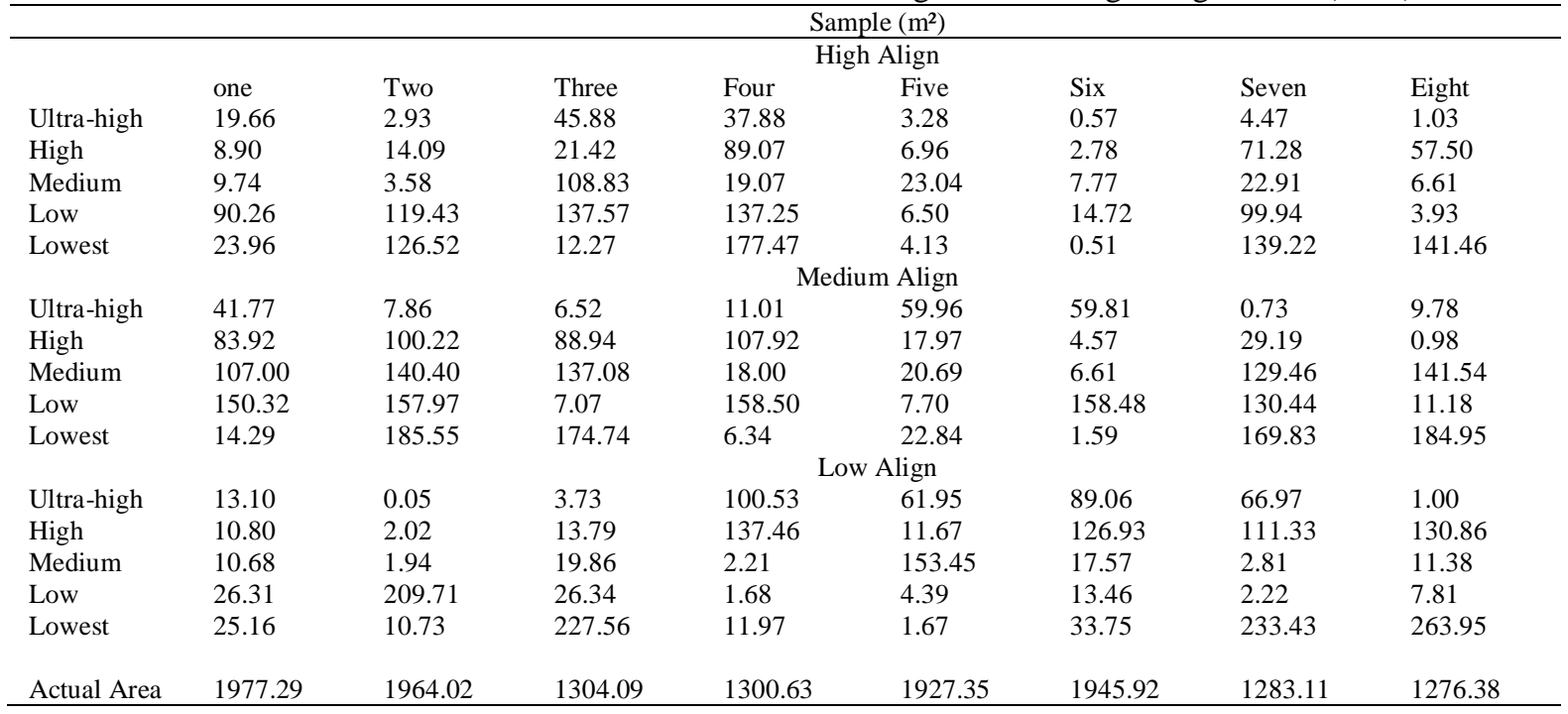




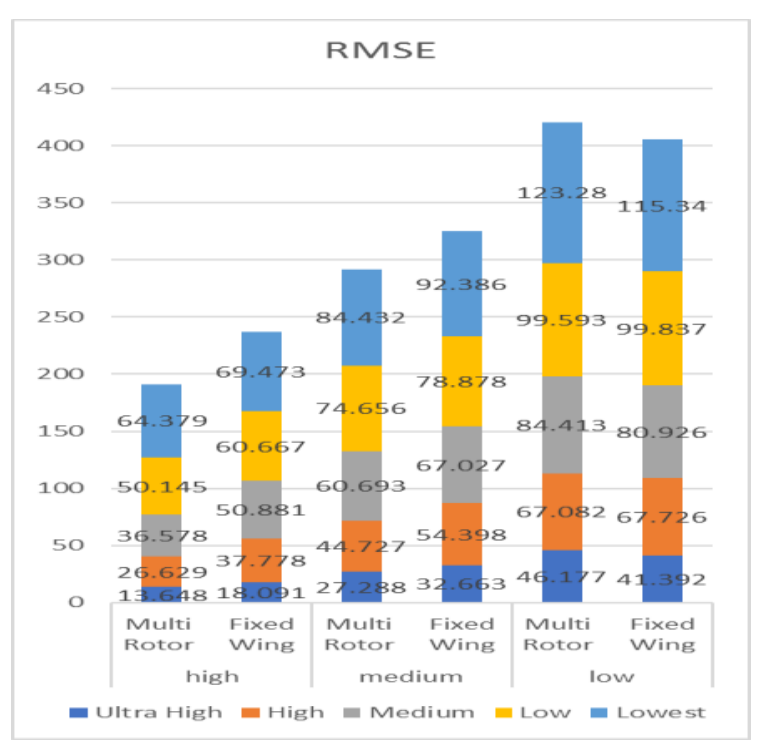

Figure 5. RMSE of Area; a) High, b) Medium, c) Low

The different between multi rotor and fixed wing illustrate in Table 2. Table 2 shows the different between multi rotor and fixed wing for ultra-high build dense records approximately $+5 \mathrm{~m}$ for all align photos. It shows that the RMSE of multi rotor UAV is lower than the RMSE of fixed wing UAV for high and medium align photos, except for low. Other than that, the high in align photo provides better result than those for medium and low, as the RMSE appears to be at its lowest.

Table 2. Different RMSE between Multi Rotor and Fixed Wing

\begin{tabular}{cccc}
\hline & High $\left(\mathrm{m}^{2}\right)$ & Medium $\left(\mathrm{m}^{2}\right)$ & Low $\left(\mathrm{m}^{2}\right)$ \\
\hline Ultra-High & 4.443 & 5.375 & 4.785 \\
High & 11.149 & 9.671 & 0.644 \\
Medium & 14.303 & 6.334 & 3.487 \\
Low & 10.522 & 4.222 & 0.244 \\
Lowest & 5.093 & 7.953 & 7.935 \\
\hline
\end{tabular}

\section{CONCLUSION}

Based on the RMSE results, the ultra-high of build dense cloud from high in align photo provides the best results as the RMSE is $13.648 \mathrm{~m}^{2}$ for multi rotor UAV and $18.091 \mathrm{~m}^{2}$ for fixed wing UAV. Overall, the measurements from the multi rotor UAV are closer with the measurements from the existing layout plans, when compared with the measurements from fixed wing UAV. For future studies, it is hoped that different types of UAV can be used in image acquisition with varied sensors, weights, and cameras. The different methods of building extraction can also be applied in the future studies to increase the accuracy of building footprint.

\section{ACKNOWLEDGEMENTS}

Faculty of Architecture, Planning, and Surveying UniversitiTeknologi MARA (UiTM), Research Management Institute (RMi) and Ministry of Higher Education (MOHE) are greatly acknowledged for providing the fund BESTARI 600-IRMI/MyRA 5/3/BESTARI (001/2017) to enable this research to be carried out. The authors would also like to thank the people who were directly or indirectly involved in this research.

\section{REFERENCES}

[1] F. Agüera-Vega et al., "Reconstruction of extreme topography from UAV structure from motion photogrammetry," Meas. J. Int. Meas. Confed., vol. 121, pp. 127-138, 2018.

[2] A. F. F. Abu Hanipah and K. N. Tahar, "Development of the 3D dome model based on a terrestrial laser scanner," Int. J. Build. Pathol. Adapt., vol. 36, no. 2, pp. 122-136, 2018. 
[3] . Brovelli, G. Zamboni, M. A. Brovelli, and G. Zamboni, "A New Method for the Assessment of Spatial Accuracy and Completeness of OpenStreetMap Building Footprints," ISPRS Int. J. Geo-Information, vol. 7, no. 289, pp. 125, Jul. 2018.

[4] F. Nex and F. Remondino, "UAV for 3D mapping applications: A review," Applied Geomatics, vol. 6, no. 1. pp. 115, 08-Mar-2014.

[5] X. Tong et al., "Integration of UAV-based photogrammetry and terrestrial laser scanning for the three-dimensional mapping and monitoring of open-pit mine areas," Remote Sens., vol. 7, no. 6, pp. 6635-6662, 2015.

[6] L. G. El-Deen Taha and R. E. Ibrahim, "New object based model for automatic building extraction by integrating LiDAR point clouds and LiDAR derived layers," Int. J. Circuits, Syst. Signal Process., vol. 10, pp. 306-315, 2016.

[7] İ. Büyüksalih, "Building Zone Regulation Compliance Using LIDAR Data: Real-Life Tests in İstanbul," Int. J. Environ. Geoinformatics, vol. 3, no. 1, pp. 48-55, 2018.

[8] N.-D. Hoang, "Detection of Surface Crack in Building Structures Using Image Processing Technique with an Improved Otsu Method for Image Thresholding," Adv. Civ. Eng., vol. 2018, pp. 1-10, Apr. 2018.

[9] G. Forlani, E. Dall'Asta, F. Diotri, U. M. di Cella, R. Roncella, and M. Santise, "Quality assessment of DSMs produced from UAV flights georeferenced with on-board RTK positioning," Remote Sens., vol. 10, no. 311, pp. 1-22, 2018.

[10] F.-J. Mesas-Carrascosa, F. Agüera-Vega, P. Martínez-Carricondo, F.-J. Pérez-Porras, F. Carvajal-Ramírez, and A. García-Ferrer, "Assessment of UAV-photogrammetric mapping accuracy based on variation of ground control points," Int. J. Appl. Earth Obs. Geoinf., vol. 72, pp. 1-10, 2018.

[11] J. V. Marcaccio, C. E. Markle, and P. Chow-Fraser, "Use of fixed-wing and multi-rotor unmanned aerial vehicles to map dynamic changes in a freshwater marsh," J. Unmanned Veh. Syst., vol. 4, no. 3, pp. 193-202, Sep. 2016.

[12] T. N. Tonkin and N. G. Midgley, "Ground-control networks for image based surface reconstruction: An investigation of optimum survey designs using UAV derived imagery and structure-from-motion photogrammetry," Remote Sens., vol. 8, no. 786, pp. 1-8, 2016.

[13] A. Eltner, P. Baumgart, H. G. Maas, and D. Faust, "Multi-temporal UAV data for automatic measurement of rill and interrill erosion on loess soil," Earth Surf. Process. Landforms, vol. 40, no. 6, pp. 741-755, 2015.

[14] V. Otero et al., "Managing mangrove forests from the sky: Forest inventory using field data and Unmanned Aerial Vehicle (UAV) imagery in the Matang Mangrove Forest Reserve, peninsular Malaysia," For. Ecol. Manage., vol. 411, pp. 35-45, 2018.

[15] N. F. H. Jumaat, B. Ahmad, and H. S. Dutsenwai, "Land cover change mapping using high resolution satellites and unmanned aerial vehicle," in IOP Conference Series: Earth and Environmental Science, 2018, vol. 169, no. 1, p. 012076.

[16] P. Gabrlik, "The use of direct georeferencing in aerial photogrammetry with micro UAV," in IFAC-PapersOnLine, 2015, vol. 28, no. 4, pp. 380-385.

[17] G. Nugroho, Z. Taha, T. S. Nugraha, and H. Hadsanggeni, "Development of a Fixed Wing Unmanned Aerial Vehicle (UAV) for Disaster Area Monitoring and Mapping," J. Mechatronics, Electr. Power, Veh. Technol., vol. 6, no. 2 , p. $83,2015$.

[18] J. A. Gonçalves and R. Henriques, "UAV photogrammetry for topographic monitoring of coastal areas," ISPRS J. Photogramm. Remote Sens., vol. 104, pp. 101-111, 2015.

[19] F. Siddiqui, S. Teng, M. Awrangjeb, and G. Lu, "A Robust Gradient Based Method for Building Extraction from LiDAR and Photogrammetric Imagery,” Sensors, vol. 16, no. 7, p. 1110, Jul. 2016.

[20] J. Koci, B. Jarihani, R. Sidle, J. X. Leon, S. Wilkinson, and R. Bartley, “Assessment of UAV and Ground-Based Structure from Motion with Multi-View Stereo Photogrammetry in a Gullied Savanna Catchment," ISPRS Int. J. Geo-Information, vol. 6, no. 11, p. 328, 2017.

[21] S. Jabari, F. Fathollahi, A. Roshan, and Y. Zhang, "Improving UAV imaging quality by optical sensor fusion: an initial study,” Int. J. Remote Sens., vol. 38, no. 17, pp. 4931-4953, Sep. 2017.

[22] R. Mlambo, I. H. Woodhouse, F. Gerard, and K. Anderson, "Structure from motion (SfM) photogrammetry with drone data: A low cost method for monitoring greenhouse gas emissions from forests in developing countries," Forests, vol. 8, no. 3, p. 68, 2017.

[23] N. Long, B. Millescamps, B. Guillot, F. Pouget, and X. Bertin, "Monitoring the topography of a dynamic tidal inlet using UAV imagery," Remote Sens., vol. 8, no. 5, p. 387, 2016.

[24] L. Javernick, J. Brasington, and B. Caruso, "Modeling the topography of shallow braided rivers using Structurefrom-Motion photogrammetry," Geomorphology, vol. 213, pp. 166-182, 2014.

[25] K. N. Tahar, "Multi Rotor Uav At Different Altitudes For Slope Mapping Studies," ISPRS - Int. Arch. Photogramm. Remote Sens. Spat. Inf. Sci., vol. XL-1/W4, pp. 9-16, Aug. 2015. 


\section{BIOGRAPHIES OF AUTHORS}
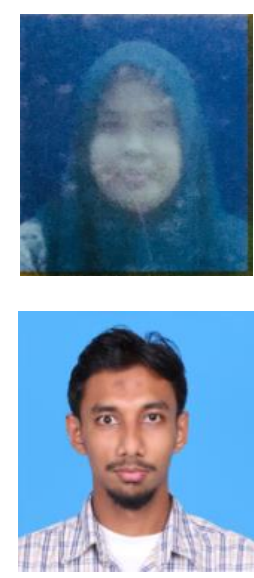

Nabila Ismail is currently employee in GIS company inn Malaysia. She obtained her Bachelor of Surveying Science and Geomatics (Hons) from Universiti Teknologi MARA, Malaysia in 2018.

Khairul Nizam Tahar is currently a Senior Lecturer in Centre of Studies for Surveying Science and Geomatics, Faculty of Architecture Planning and Surveying, Universiti Teknologi MARA (UiTM), Malaysia. He obtained his Dip Geomatic Science (GIS), BSc (Hons) Surveying Science and Geomatics and MSc (GIS) degrees from Department of Surveying Science and Geomatics, UiTM, Malaysia in 2005, 2007 and 2008 respectively and $\mathrm{PhD}$ degree from Universiti Teknologi Malaysia (UTM), Malaysia in 2013. His major research includes geomatics field such as aerial photogrammetry and close range photogrammetry especially on UAV applications. He has published 1 book, 1 book chapter and over 60 articles in journals and conference proceedings. $\mathrm{He}$ also received a number of research grants from university and ministry. He conducts a few research as the principal investigator and co-investigator on UAV positioning, building footprint extraction using UAV, rapid airborne geospatial mapping system and automatic counting of shipping containers using UAV. 